Technical Note

\title{
Establishment of diagnostic reference levels arising from common CT examinations in Semnan County, Iran
}

\author{
Daryoush KHORAMIAN ${ }^{1, a}$, Soroush SISTANI ${ }^{1}$, Peyman HEJAZI ${ }^{1}$ \\ ${ }^{I}$ Department of Medical Physics, Student Research Committee, Faculty of Medicine, Semnan University of Medical Sciences \\ ${ }^{a}$ E-mail address: daryoushkhoramian@gmail.com
}

(received 19 August 2018; revised 1 December 2018; accepted 19 December 2018)

\begin{abstract}
Objective: The literature has approved that the use of the concept of diagnostic reference level (DRL) as a part of an optimization process could help to reduce patient doses in diagnostic radiology comprising the Computed Tomography (CT) examinations. There are four public/governmental CT centers in the province (Semnan, Iran) and, to our knowledge, after about 12 years since the launch of the first CT scanner in the province there is no dosimetry information on those CT scanners. The aim of this study was to evaluate CT dose indices with the aim of the establishment of the DRL for head, chest, cervical spine, and abdomen-pelvis examinations.

Methods: Scan parameters of 381 patients were collected during two months from 4 CT scanners. The CT dose index (CTDI) was measured using a calibrated ionization chamber on two cylindrical poly methyl methacrylate (PMMA) phantoms. For each sequences, weighted CTDI (CTDIw), volumetric CTDI (CTDIv) and dose length product (DLP) were calculated. The 75th percentile was proposed as the criterion for DRL values.

Results: Proposed DRL (CTDIw, CTDIv, DLP) for the head, chest, cervical spine, and abdomen-pelvis were (46.1 mGy, $46.1 \mathrm{mGy}, 723 \mathrm{mGy} \times \mathrm{cm}),(13.8 \mathrm{mGy}, 12.0 \mathrm{mGy}, 377 \mathrm{mGy} \times \mathrm{cm}),(40.0 \mathrm{mGy}, 40.0 \mathrm{mGy}, 572 \mathrm{mGy} \times \mathrm{cm})$ and (14.9 mGy, $12.1 \mathrm{mGy}, 524 \mathrm{mGy} \times \mathrm{cm})$, respectively.

Conclusion: Comparison with the others results from the other countries indicates that the head, chest and abdomenpelvis scans in our region are lower or in the range of the other studies investigated in terms of dose. In the case of cervical spine scanning it's necessary to review and regulate scan protocols to reach acceptable dose levels.
\end{abstract}

Key words: Diagnostic Reference Level; CT scanning; CT dose indices.

\section{Introduction}

Computed Tomography (CT) scanning with the ability to perform scans in a very short time alongside high image quality has become a common imaging modality in diagnostic radiology [1]. The use of this imaging modality dramatically has increased in recent years by the emergence of multidetector CT scanners (MDCT), about 12-fold in the UK and more than 20-fold in the USA in last 25 years [2].

Although CT became one of the most useful x-ray based imaging modalities, relatively high doses to the patients during CT procedures (about 1 to $24 \mathrm{mSv}$ ) have to be considered [3]. CT is responsible for over 44 percent of the global medical radiation to population worldwide, thus $\mathrm{CT}$ scan stands as one of the high radiation dose imaging techniques [4].

According to ALARA principle (as low as reasonably achievable), all medical ionization radiation-based imaging equipments should be operating at optimum performance [5]. As a part of optimization process, Diagnostic Reference Level (DRL) has been introduced by the International Commission on Radiological Protection in ICRP publication no.73 by 1996 for common diagnostic procedures and implemented in various regions and countries [6-8], in order to decrease patient doses during medical imaging procedures comprising CT examinations [6]. The DRL is a useful tool for optimization [9] and to managing patient dose [10]. The use of DRL in the UK has led to about $50 \%$ reduction in the radiation dose in typical radiographic examinations in a period of 15 years [11].

The DRL is usually set at 75 th percentile of measured dose in standard phantom or patient. If the level is set based on patients' measurements, the mean of patients' heights and weights should be nearly identical. A reduction in the number of measurements is the advantage of the use of the phantom. Although phantom does not fully match the characteristics of real patients, the use of phantom measurements decreases the number of measurements by one or two for each procedure. So in multicenter studies, phantom measurements are recommended $[6,7]$.

DRL does not determine dose constraint for an individual patient, the goal of DRL is the delineation of a level of doses for special procedures higher than which are an unusual/ unnecessary doses received by the patients [7]. If the dose arising from an x-ray based imaging modality, e.g. CT scan, in 
a certain anatomical regions is above national (NDRL) or Local DRL (LDRL), the scan parameters should be reviewed and revised. Typically, the establishment of DRL is a part of a quality assurance programme and the proposed levels are advisory $[6,12,13]$.

There are four public/governmental hospitals in Semnan province in Iran (1 CT scanner per 150,000 individuals). The first CT scanner was installed in 2007 and three other scanners were installed in 2009, 2012 and 2015. To our knowledge, after about ten years of the first installed CT scanner, there is no dosimetry information on these CT scanners. Thus, in light of the aforementioned views, the goal of this study was to provide data from CT scan procedures and the establishment of DRL of the head, cervical spine, chest, and abdomen-pelvis CT procedures in adult patients in Semnan province in Iran.

\section{Material and Method}

\section{Survey framework}

This study was carried out using questionnaires to collect scan details of the most frequent CT examinations of the averagesize adult patients referring to public/governmental hospitals in the Semnan province of Iran. Questionnaires were sent to the hospitals and asked the centers to complete each of them for an average-size patient. The information on the questionnaire included: hospital name, CT scanner model, year of installation, patients' age, patients' height and weight for the calculation of Body Mass Index (BMI), tube potential, tube current-time, pitch factor, total collimation, scan length and anatomical region of the scan. The CTDI values were measured with the average scan parameters for each scan with the aid of appropriate dosimeter and phantoms. The CTDIw, CTDIv and the DLP values were calculated. The 75th percentile values of the calculated CTDIw, CTDIv and DLP were proposed as DRL in each sequence.

\section{CT scanners}

In this study, four CT scanners, including one single slice (Siemens Emotion), one 2-MDCT (Siemens Emotion DUO) and two 16-MDCT (Toshiba Aquilion, Toshiba Activion) were investigated.

\section{Dose measurement}

Table 1. Details of scanners participated in this study.

\begin{tabular}{|c|c|c|c|c|c|c|c|}
\hline Scanner & X-ray tube & $\begin{array}{c}\begin{array}{c}\text { Gantry aperture } \\
(\mathrm{cm})\end{array} \\
\end{array}$ & $\begin{array}{c}\text { Focal spot } \\
(\mathrm{mm})\end{array}$ & $\begin{array}{c}\text { Total filtration } \\
\text { (mm Al equivalent) }\end{array}$ & Detector & $\begin{array}{c}\text { Number of } \\
\text { detectors in z-axis }\end{array}$ & $\begin{array}{c}\text { Tube current } \\
\text { range (step) }\end{array}$ \\
\hline Siemens Emotion & Siemens Dura 302-MV & 70 & $\begin{array}{l}0.4 \times 0.8 \\
0.7 \times 0.8\end{array}$ & $6.4(80 \mathrm{kVp})$ & $\begin{array}{l}\text { Ultra-Fast } \\
\text { Ceramic }\end{array}$ & single slice & $30-240(10 \mathrm{~mA})$ \\
\hline Siemens Emotion Duo & Siemens Dura 352 & 70 & $\begin{array}{l}0.4 \times 0.8 \\
0.7 \times 0.8\end{array}$ & $6.4(80 \mathrm{kVp})$ & $\begin{array}{l}\text { Ultra-Fast } \\
\text { Ceramic }\end{array}$ & 2 & $30-240(1 \mathrm{~mA})$ \\
\hline Toshiba Aquilion & Toshiba Megacool & 72 & $\begin{array}{l}0.8 \times 0.9 \\
1.4 \times 1.6\end{array}$ & $\begin{array}{l}1.5-10 \text { (wedge } \\
\text { dependent) }\end{array}$ & Solid state & 40 & $50-500(10 \mathrm{~mA})$ \\
\hline Toshiba Activion & CXB-400C & 72 & $\begin{array}{l}0.9 \times 0.7 \\
1.4 \times 1.4\end{array}$ & $11(120 \mathrm{kVp})$ & Solid stare & 28 & $10-300$ \\
\hline
\end{tabular}

The standardized scattering media for CT dose measurements approved by FDA are two cylindrical poly-methylmethacrylate (PMMA) phantoms [14]. All measurements were performed on PMMA head and body phantoms with the length of $15 \mathrm{~cm}$ and the diameter of 16 and $32 \mathrm{~cm}$, respectively. These phantoms contain one hole in the center and four holes at the periphery near the surface $(1 \mathrm{~cm}$ below the surface). The integrated absorbed dose $(\mathrm{D}(\mathrm{z}))$ along the $\mathrm{z}$-direction/patient direction was measured from one axial scan by a calibrated ionization chamber (model DCT-10 - RTI Electronics, Sweden) with an active length of $100 \mathrm{~mm}$ using Barracuda multi-meter (RTI Electronics, Sweden). The ionization chamber plugged in at the intended hole and the other holes filled by PMMA rods. This procedure was done for each of five holes. CTDI $_{100}$-which refers to the active length of the ionization chamber- was calculated by dividing the measured absorbed dose by the nominal total beam width $(\mathrm{N} \times \mathrm{T})$ according to the following formula:

$\operatorname{CTDI}_{100}=\frac{1}{N T} \int_{-50 \mathrm{~mm}}^{50 \mathrm{~mm}} D(z) d z$ Eq. 1

For each sequences the CTDIw, CTDIv and DLP were calculated using Equations 2, 3 and 4:

$$
\begin{aligned}
& C T D I_{w}=\frac{1}{3}\left(C T D I_{100, c}\right)+\frac{2}{3}\left(C T D I_{100, p}\right) \\
& C T D I_{v}=C T D I_{w} / \text { pitch } \\
& D L P=C T D I_{v} \times \text { scan length }
\end{aligned}
$$$$
\text { Eq. } 2
$$

Eq. 4

\section{Results}

During a period of 2 months, data from 381 patients undergoing routine head, cervical spine, chest and abdomenpelvis scans were collected in the four public CT centers of Semnan province, Iran. From total examinations, 117 patients (31\%) went for head scan which was the most frequent exam. Other $98(26 \%), 86(22 \%)$, and $80(21 \%)$ patients were had routine chest, cervical spine and abdomen-pelvis scans performed on them, respectively. It's notable that the number of the patients were 425, patients with normal BMI $(21<\mathrm{BMI}<24.7)$ being included and the over/under BMI values being rejected. 
Table 2. Scan parameters and patient characteristics investigated in this study.

\begin{tabular}{|c|c|c|c|c|c|c|c|c|c|}
\hline Anatomical site & CT scanner & No. of patients & Age (years) & BMI & $\mathbf{k V p}$ & mAs & Pitch & $\begin{array}{c}\text { Total collimation } \\
(\mathbf{m m})\end{array}$ & $\begin{array}{c}\text { Scan length } \\
(\mathrm{cm}) *\end{array}$ \\
\hline \multirow{4}{*}{ Head } & Siemens Emotion & 24 & $43 \pm 13$ & 22.4 & 130 & 170 & Axial & 8 & $14.0 \pm 1.1$ \\
\hline & Siemens Emotion Duo & 23 & $52 \pm 21$ & 22.5 & 110 & 160 & Axial & 8 & $15.1 \pm 1.3$ \\
\hline & Toshiba Aquilion & 32 & $57 \pm 19$ & 21.8 & 120 & 225 & Axial & 5 & $16.1 \pm 1.4$ \\
\hline & Toshiba Activion & 38 & $49 \pm 16$ & 23.8 & 120 & 150 & Axial & 8 & $13.0 \pm 1.3$ \\
\hline \multirow{4}{*}{ Chest } & Siemens Emotion & 21 & $55 \pm 11$ & 21.8 & 130 & 100 & 1.8 & 20 & $30.0 \pm 2.1$ \\
\hline & Siemens Emotion Duo & 21 & $48 \pm 16$ & 21.9 & 130 & 140 & 1 & 20 & $31.6 \pm 2.3$ \\
\hline & Toshiba Aquilion & 30 & $50 \pm 17$ & 23.8 & 120 & 160 & 1.4 & 20 & $30.1 \pm 2.4$ \\
\hline & Toshiba Activion & 26 & $43 \pm 12$ & 24.0 & 120 & 75 & 1.2 & 8 & $30.6 \pm 3.3$ \\
\hline \multirow{4}{*}{ Cervical spine } & Siemens Emotion & 20 & $40 \pm 11$ & 24.2 & 130 & 170 & Axial & 8 & $10.3 \pm 0.9$ \\
\hline & Siemens Emotion Duo & 25 & $39 \pm 7$ & 23.8 & 130 & 130 & Axial & 8 & $15.8 \pm 1.6$ \\
\hline & Toshiba Aquilion & 21 & $50 \pm 10$ & 23.1 & 120 & 150 & Axial & 5 & $14.1 \pm 2.9$ \\
\hline & Toshiba Activion & 20 & $51 \pm 18$ & 21.5 & 120 & 188 & Axial & 8 & $14.8 \pm 1.5$ \\
\hline \multirow{4}{*}{$\begin{array}{l}\text { Abdomen- } \\
\text { Pelvis }\end{array}$} & Siemens Emotion & 20 & $55 \pm 21$ & 24.2 & 130 & 130 & 1.2 & 16 & $46.9 \pm 2.0$ \\
\hline & Siemens Emotion Duo & 20 & $51 \pm 18$ & 24.1 & 130 & 160 & 1 & 20 & $43.3 \pm 2.5$ \\
\hline & Toshiba Aquilion & 20 & $49 \pm 12$ & 23.6 & 120 & 170 & 1.4 & 20 & $44.2 \pm 4.7$ \\
\hline & Toshiba Activion & 20 & $44 \pm 19$ & 23.4 & 120 & 113 & 1.2 & 8 & $41.2 \pm 3.6$ \\
\hline
\end{tabular}

Table 3. The mean values of the CTDIw, CTDIv and DLP values.

\begin{tabular}{|c|c|c|c|c|}
\hline Anatomical site & CT scanner & $\begin{array}{c}\text { CTDIw } \\
(\mathrm{mGy})\end{array}$ & $\begin{array}{l}\text { CTDIv } \\
\text { (mGy) }\end{array}$ & DLP $(\mathrm{mGy} \times \mathrm{cm})$ \\
\hline \multirow{6}{*}{ Head } & Siemens Emotion & 34.3 & 34.3 & 480 \\
\hline & Siemens Emotion Duo & 31.6 & 31.6 & 477 \\
\hline & Toshiba Aquilion & 49.9 & 49.9 & 804 \\
\hline & Toshiba Activion & 33.1 & 33.1 & 430 \\
\hline & Mean of four scanners & 37.2 & 37.2 & 547 \\
\hline & DRL & 46.1 & 46.1 & 723 \\
\hline \multirow{6}{*}{ Chest } & Siemens Emotion & 10.6 & 5.9 & 177 \\
\hline & Siemens Emotion Duo & 12.7 & 12.7 & 401 \\
\hline & Toshiba Aquilion & 14.1 & 10.1 & 304 \\
\hline & Toshiba Activion & 6.9 & 5.7 & 175 \\
\hline & Mean of four scanners & 11.1 & 8.6 & 264 \\
\hline & DRL & 13.8 & 12.0 & 377 \\
\hline \multirow{6}{*}{ Cervical spine } & Siemens Emotion & 40.4 & 40.4 & 416 \\
\hline & Siemens Emotion Duo & 34.9 & 34.9 & 552 \\
\hline & Toshiba Aquilion & 36.1 & 36.1 & 510 \\
\hline & Toshiba Activion & 39.2 & 39.2 & 579 \\
\hline & Mean of four scanners & 37.7 & 37.7 & 515 \\
\hline & DRL & 40.0 & 40.0 & 572 \\
\hline \multirow{6}{*}{ Abdomen- Plevis } & Siemens Emotion & 11.8 & 9.9 & 462 \\
\hline & Siemens Emotion Duo & 12.3 & 12.3 & 533 \\
\hline & Toshiba Aquilion & 15.8 & 11.2 & 497 \\
\hline & Toshiba Activion & 11.4 & 9.5 & 391 \\
\hline & Mean of four scanners & 12.8 & 10.7 & 471 \\
\hline & DRL & 14.9 & 12.1 & 524 \\
\hline
\end{tabular}

All the procedures were performed without the administration of a contrast agent. All the head and cervical spine scans were axial and all the chest and abdomen-pelvis scans were spiral with a pitch factor of 1 and above. Table 2 represents scan parameters and patient characteristics participating in present study.
On all CT scanners, the $\mathrm{CTDI}_{100}$ were measured using the head and body PMMA phantoms and $100 \mathrm{~mm}$-length ionization chamber, and the CTDIw, CTDIv and DLP were calculated. The 75 th percentile values were proposed as DRL. Table 3 presents mean value of the CTDIw, CTDIv and DLP on each scanner, in addition to the mean value of all scanners and the proposed DRL values. 

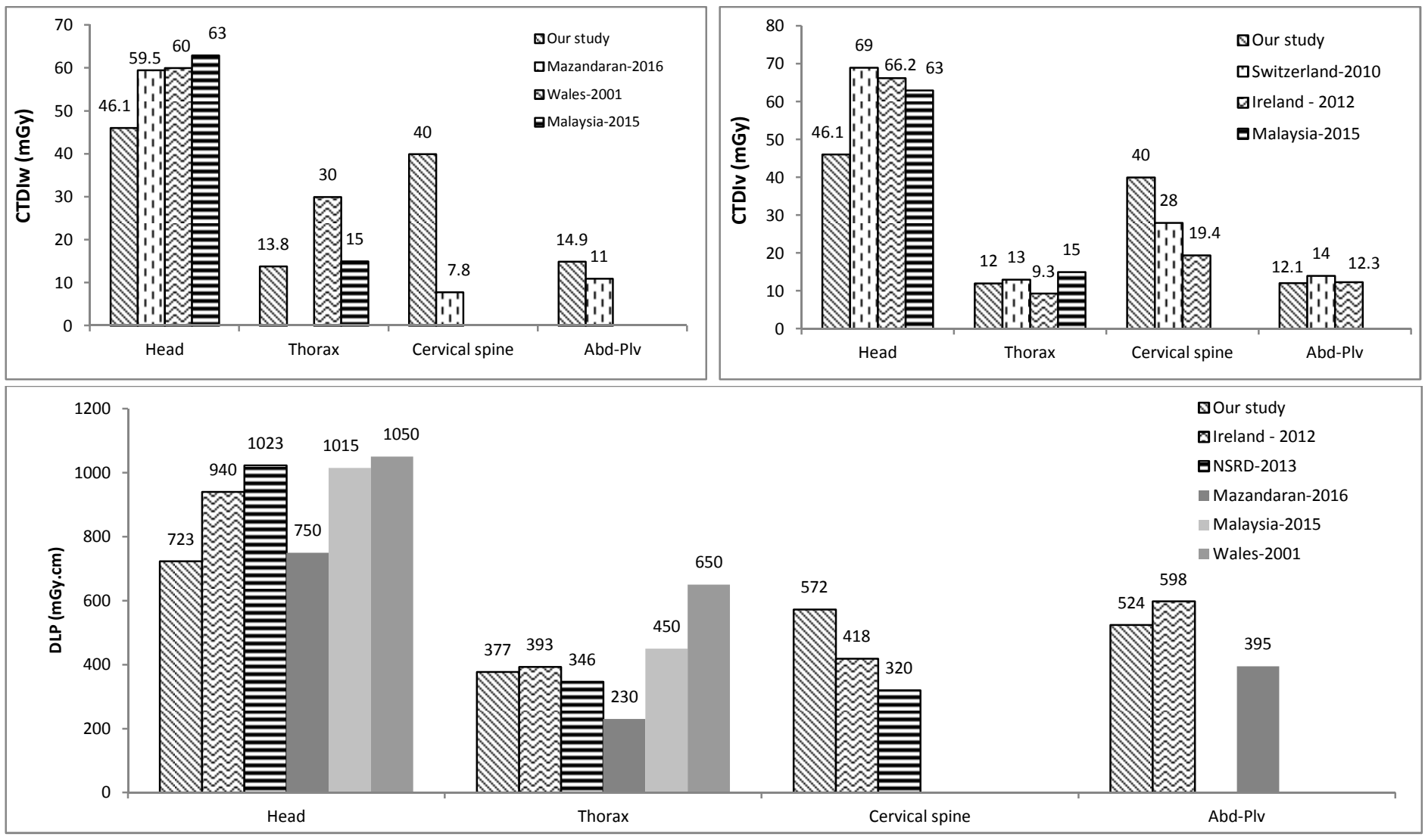

Figure 1. The DRL values of the CTDIw, CTDIv and DLP. Comparison between scan region and the other works reviewed.

\section{Discussion}

In this study, we did a calculation of the CTDIw, CTDIv and DLP to assess doses arising from four CT examinations to establish the DRL of the head, cervical spine, chest, and abdomen-pelvis examinations in Semnan province, Iran.

\section{Inter-hospitals comparison}

As the results indicate dose indices vary from hospital to hospital which is due to using different scan parameters for the same scan region.

Head Scan: The CTDIw and CTDIv ranged from $33.1 \mathrm{mGy}$ (on Toshiba Activion) to $44.9 \mathrm{mGy}$ (on Toshiba Aquilion). The DLP values ranged from $430 \mathrm{mGy} \times \mathrm{cm}$ (on Toshiba Activion) to $804 \mathrm{mGy} \times \mathrm{cm}$ (on Toshiba Aquilion). The higher values of the CTDIw, CTDIv and DLP on the Toshiba Aquilion result from the use of higher tube current-time $(225 \mathrm{mAs})$ on this scanner (Table 3).

Chest Scan: The CTDIw ranged from $6.9 \mathrm{mGy}$ (on Toshiba Activion) to $14.1 \mathrm{mGy}$ (on Toshiba Aquilion). The higher dose from Toshiba Aquilion is due to the use of higher tube currenttime (160 mAs). The CTDIv ranged from $5.7 \mathrm{mGy}$ (on Toshiba Activion) to $12.7 \mathrm{mGy}$ (Siemens Emotion Duo). DLP ranged from $175 \mathrm{mGy} \times \mathrm{cm}$ (on Toshiba Activion) to $401 \mathrm{mGy} \times \mathrm{cm}$ (Siemens Emotion Duo) (Table 3). Although CTDIw on Toshiba Aquilion is higher than the other CT scanners, the use of lower pitch on Siemens Emotion Duo (pitch=1) results in highest CTDIv and DLP on this scanner.
Cervical spine Scan: The CTDIw and CTDIv ranged from 34.9 mGy (Siemens Emotion Duo) to $40.4 \mathrm{mGy}$ (Siemens Emotion). The range of the DLP varies from $416 \mathrm{mGy} \times \mathrm{cm}$ (on Siemens Emotion) to $579 \mathrm{mGy} \times \mathrm{cm}$ (on Toshiba Activion) (Table 3). The highest values of the CTDIw and CTDIv belong to cervical spine scans due to using high tube current-time (170 $\mathrm{mAs})$ and tube potential $(130 \mathrm{kVp})$. Also, using of less scan length on this scanner results in lowest DLP $(416 \mathrm{mGy} \times \mathrm{cm})$.

Abdomen-Pelvis Scan: The CTDIw ranged from $11.4 \mathrm{mGy}$ (on Toshiba Activion) to $15.8 \mathrm{mGy}$ (on Toshiba Aquilion). The range of the CTDIv varies from $9.5 \mathrm{mGy}$ (on Toshiba Activion) to $12.3 \mathrm{mGy}$ (Siemens Emotion Duo). The DLP ranged from $391 \mathrm{mGy} \times \mathrm{cm}$ (on Toshiba Activion) to 533 $\mathrm{mGy} \times \mathrm{cm}$ (Siemens Emotion Duo) (Table 3). Although the highest value of the CTDIw was for Toshiba Aquilion (15.8 mGy), using higher pitch relative to Siemens Emotion Duo (1.4 vs. 1) causes lower CTDIv and DLP values.

\section{Comparison with the other studies}

Figure 1 reveals that the DRL values of the CTDIw, CTDIv and DLP for the head scan are lower than the other studies which have been investigated in the present study. For the chest scan, the CTDIw was lower than the other studies, whereas the CTDIv is higher than the Ireland [8] report and lower than the Switzerland [1] and Malaysia DRLs. Also, the DLP for chest scan is higher than the Mazandaran [16] and Netherlands [17] and is lower than the other regions. In the case of cervical spine the CTDIw (about 5 times higher than 
Mazandaran value), CTDIv and DLP values are higher than the other reports. For the abdomen-pelvis scan, the CTDIw value is higher than Mazandaran DRL. The CTDIv is lower than Switzerland and Ireland and the DLP is lower than Ireland and higher than Wales [18] reports.

\section{Conclusion}

Beside of the fact that CT scan provides useful images and aids to physicians in diagnosing a wide range of diseases, relatively high dose from CT examinations relative to the other imaging modalities is a matter of concern. Therefore, it was important to know the magnitude of doses received by the patients. The literature shows the DRL as a part of optimization programs can help to reduce patient's doses over the time. Our study is the first dose assessment survey in the province since the installation of the first MDCT scanner in 2007.

According to our results, as anticipated, the CTDIw values are higher in the head and cervical spine scans which is due to using smaller phantom size $(16 \mathrm{~cm}$ diameter vs. $32 \mathrm{~cm}$ in body region). This argument cannot be used for CTDIv and DLP which is influenced by the pitch factor and scan length, respectively.

Our results reveal that doses from the head, chest and abdomen-pelvis scans in our region are lower or in the range of the other studies investigated. In the case of cervical spine scanning it's necessary to review and regulate scan protocols to reach an acceptable dose level. Protocol optimization was beyond of the scope of this study.

\section{References}

[1] Treier R, Aroua A, Verdun F, et al. Patient doses in CT examinations in Switzerland: implementation of national diagnostic reference levels. Radiat Prot Dosimetry. 2010;142(2-4):244-254.

[2] Mulkens T, Salgado R, Bellinck P. Dose Optimization and Reduction in CT of the Brain and Head and Neck Region. In: Tack D, Kalra M, Gevenois P (eds). Radiation Dose from Multidetector CT. Medical Radiology. Springer, Berlin, Heidelberg. 2011. p. 281306.

[3] Tack D, Gevenois P (eds). Radiation Dose from Adult and Pediatric Multidetector Computed Tomography. Springer. 2007. section 6:276.

[4] Karim MKA, Hashim S, Bradley DA, et al. Radiation doses from computed tomography practice in Johor Bahru, Malaysia. Radiat Phys Chem. 2016;121:69-74.

[5] Reiser MF, Hricak H, Knauth M. Radiation Dose from Multidetector CT. Springer-Verlag. 2012. p. 642.

[6] European Commission. Guidance on diagnostic reference levels (DRLs) for medical exposures: Radiation Protection 109. Directorate-General, Environment, Nuclear Safety and Civil Protection, European Commission; 1999. p. 26.

[7] Vassileva J, Rehani M. Diagnostic reference levels. AJR Am J Roentgenol. 2015;204(1):W1-W3.

[8] Foley SJ, McEntee MF, Rainford LA. Establishment of CT diagnostic reference levels in Ireland. Br J Radiol. 2012;85(1018):13901397.

[9] Cousins C. Changes Impacting on Radiation Protection in Medicine Since the Malaga Conference. in: Radiation Protection in Medicine: Setting the Scene for the Next Decade. Proceedings of an International Conference 3-7 December 2012, Bonn, Germany. International Atomic Energy Agency (IAEA): IAEA. 2015 p. 31-35.

[10] Zhang M, Chu C. Optimization of the radiological protection of patients undergoing digital radiography. J Digit Imaging. 2012;25(1):196-200.

[11] Hart D, Wall B. UK population dose from medical X-ray examinations. Eur J Radiol. 2004;50(3):285-291.

[12] Jurkovic S, Segota D, Posedel D, et al. Radiation Doses on CT Scanner in University Hospital Rijeka and Comparison with European Commision Dose Reference Levels. in: Knezevic Z; Krajcar Bronic I; Majer M. (eds). Proceedings of 9th Symposium of the Croatian Radiation Protection Association. 2013. p.259-264.

[13] Diagnostic reference levels in medical imaging: Review and additional advice. Annals of the ICRP. 2001;31(4):33-52.

[14] McCollough C, Cody D, Edyvean S, et al. AAPM Report No. 96. The measurement, reporting, and management of radiation dose in CT. Report of AAPM Task Group 23. 2008.

[15] Khoramian D, Sistani S. Estimation and comparison of the radiation effective dose during coronary computed tomography angiography examinations on single-source 64-MDCT and dual-source 128-MDCT. J Radiol Prot. 2017;37(4):826-836.

[16] Janbabanezhad Toori A, Shabestani-Monfared A, Deevband MR, et al. Dose Assessment in Computed Tomography Examination and Establishment of Local Diagnostic Reference Levels in Mazandaran, Iran. J Biomed Phys Eng. 2015;5(4):177-184.

[17] van der Molen A, Schilham A, Stoop P, et al. A national survey on radiation dose in CT in The Netherlands. Insights Imaging. 2013;4(3):383-890.

[18] Hiles P, Brennen S, Scott S, Davies J. A survey of patient dose and image quality for computed tomography scanners in Wales. J Radiol Prot. 2001;21(4):345-354. 